\title{
High-speed coronal rain
}

\author{
D. A. N. Müller ${ }^{1,2,3}$, A. De Groof ${ }^{4}$, V. H. Hansteen ${ }^{1,2}$, and H. Peter ${ }^{3}$ \\ 1 Institute of Theoretical Astrophysics, University of Oslo, PO Box 1029 Blindern, 0315 Oslo, Norway \\ e-mail: [Daniel.Mueller;Viggo.Hansteen]@astro.uio.no \\ 2 Center of Mathematics for Applications, University of Oslo, PO Box 1053 Blindern, 0316 Oslo, Norway \\ 3 Kiepenheuer-Institut für Sonnenphysik, Schöneckstr. 6, 79104 Freiburg, Germany \\ e-mail: peter@kis.uni-freiburg.de \\ ${ }^{4}$ Centrum voor Plasma-Astrofysica, KU Leuven, Celestijnenlaan 200B, 3001 Leuven, Belgium \\ e-mail: Anik.DeGroof@wis.kuleuven.ac.be
}

Received 8 October 2004 / Accepted 24 January 2005

\begin{abstract}
At high spatial and temporal resolution, coronal loops are observed to have a highly dynamic nature. Recent observations with SOHO and TRACE frequently show localized brightenings "raining" down towards the solar surface. What is the origin of these features? Here we present for the first time a comparison of observed intensity enhancements from an EIT shutterless campaign with non-equilibrium ionization simulations of coronal loops in order to reveal the physical processes governing fast flows and localized brightenings. We show that catastrophic cooling around the loop apex as a consequence of footpoint-concentrated heating offers a simple explanation for these observations. An advantage of this model is that no external driving mechanism is necessary as the dynamics result entirely from the non-linear character of the problem.
\end{abstract}

Key words. Sun: corona - Sun: transition region - Sun: UV radiation - Sun: magnetic fields - Sun: atmosphere

\section{Introduction}

The upper solar atmosphere, i.e. the transition region and corona, is highly complex and magnetically structured. Recent space observations, especially with the Solar and Heliospheric Observatory (SOHO) and the Transition Region And Coronal Explorer (TRACE), have revealed that coronal loops, magnetically closed structures in the upper solar atmosphere, are intrinsically dynamic, and intensity enhancements ("blobs") are often seen to propagate along these loops. Spectroscopic investigations show that these intensity variations have different signatures in UV spectral lines formed at different temperatures and exhibit Doppler shifts of $v=20-100 \mathrm{~km} \mathrm{~s}^{-1}$ (Fredvik et al. 2002). In an overview of observations of the temporal variability of active region loops with the Coronal Diagnostic Spectrometer (CDS), Kjeldseth-Moe \& Brekke (1998) report significant changes of coronal loops over a period of one hour, in particular seen in emission lines in the temperature range between $T=1-5 \times 10^{5} \mathrm{~K}$.

Recently, De Groof et al. (2004) observed propagating intensity variations in the He II $30.4 \mathrm{~nm}$ band with the ExtremeUltraviolet Imaging Telescope (EIT). The dominant part of the plasma emitting in this spectral band has temperatures of $T=6-8 \times 10^{4} \mathrm{~K}$. Plasma seen in this spectral band in higher layers of the solar atmosphere is thus considerably cooler than its surroundings. This can be the result of plasma draining from a prominence or material cooling down after a flare. However, when evidence for these two processes is lacking, the nature of these intensity variations is difficult to explain. While slow magneto-acoustic waves may in general account for propagating intensity variations, this explanation of the features observed in the EIT shutterless campaign from 11 July 2001 was ruled out by De Groof et al. (2004).

Another possible explanation which we propose in this work is the scenario of the "heating-condensation cycle" (Müller et al. 2003, 2004, hereafter referred to as Papers I and II). In these papers we showed that localized brightenings can be the result of catastrophic cooling of a loop which is predominantly heated at the footpoints. The heating leads to an evaporation of plasma into the coronal loop which then cools rapidly due to a loss of thermal equilibrium. The confined region of "condensed" plasma subsequently falls down under the effect of gravity in the form of a cool plasma blob. In Paper II we described the evolution, speed and spectral signature of these traveling condensation regions, and pointed out a possible connection to the recent EIT observations. Compared to the loops described in Paper II, the loop structure seen in the data from the EIT shutterless campaign is larger (with an estimated loop length of about $L=300 \mathrm{Mm}$ compared to the $L=100 \mathrm{Mm}$ models of Paper II) and the observed blob velocities are higher. Even so, the general features of the model encouraged us to carry out new simulations with changes suggested by the EIT observations. For the models presented here, we adopted the loop geometry inferred from the EIT data and 
also used a higher heating rate to reach apex temperatures of around $3 \mathrm{MK}$ as suggested, e.g., by TRACE observations of large active region loops.

\section{Numerical model}

We use the same numerical model as in Paper I, and the reader is referred to this work and to Hansteen (1993) for details. Our code solves the one-dimensional time-dependent hydrodynamic equations for mass, momentum and energy conservation, coupled with the ionization rate equations for several elements and self-consistent radiative losses. The plasma is assumed to be effectively thin, and the radiative losses are due to collisional excitation of the various ions comprising the plasma, in addition to thermal bremsstrahlung. Thermal conduction, radiative losses and a coronal heating term are included in the energy equation. In the radiative losses the elements hydrogen, helium, carbon, oxygen, silicon, neon, and iron are included. While some of the metals are treated by assuming ionization equilibrium and then deriving an a priori radiative loss curve as a function of electron temperature, radiative losses from the ions specifically mentioned in this study, i.e. losses from hydrogen, helium, carbon and oxygen, are computed consistently with full time-dependent rate equations. We consider a loop of low- $\beta$ plasma and assume that the loop has a constant cross section.

\subsection{Loop heating}

The energy input into the coronal loop is parametrized by specifying the energy flux at the footpoints of the loop, $F_{m 0}$, and assuming a mechanical energy flux that is constant up to a height $z_{1}$ and then decreases exponentially for $z \geq z_{1}$ as

$$
F_{m}(z)=F_{m 0} \exp \left[-\left(z-z_{1}\right) / H_{m}\right]
$$

with a damping length $H_{m}$ (the same parametrization as the one used in Papers I and II). In the models presented below, we will vary $H_{m}$ between 2 and $30 \mathrm{Mm}$ for a loop of $L=300 \mathrm{Mm}$ length. For the mechanical energy flux we use $F_{m 0}=c \times 10^{4} \mathrm{~W} \mathrm{~m}^{-2}$ with the normalization constant $c=$ $1 /\left(1-\exp \left[-\left(L / 2-z_{1}\right) / H_{m}\right]\right)$ and set $z_{1}=1.75 \mathrm{Mm}$. The normalization constant ensures that the total energy input into the loop is constant, irrespective of the damping length $H_{m}$. The heating rate, i.e. the energy deposition per unit time and unit volume, is given by the divergence of the energy flux, $Q_{m}=-\nabla F_{m}$. With the damping length $H_{m}$ we can control whether the heating is concentrated near the footpoints or is more evenly distributed along the loop. The energy flux we use corresponds to the estimate of Withbroe \& Noyes (1977) for the total coronal energy loss for active regions (for quiet sun regions, these authors estimate a value of $300 \mathrm{~W} \mathrm{~m}^{-2}$ ).

An exponentially decaying heating function was first suggested by Serio et al. (1981) and seems to be supported by recent observations (Aschwanden et al. 2000, 2001) as well as by numerical simulations of Gudiksen \& Nordlund (2002). Aschwanden et al. (2001) find that a damping length $H_{m}=$ $12 \pm 5 \mathrm{Mm}$ yields the best fit of hydrostatic models to TRACE

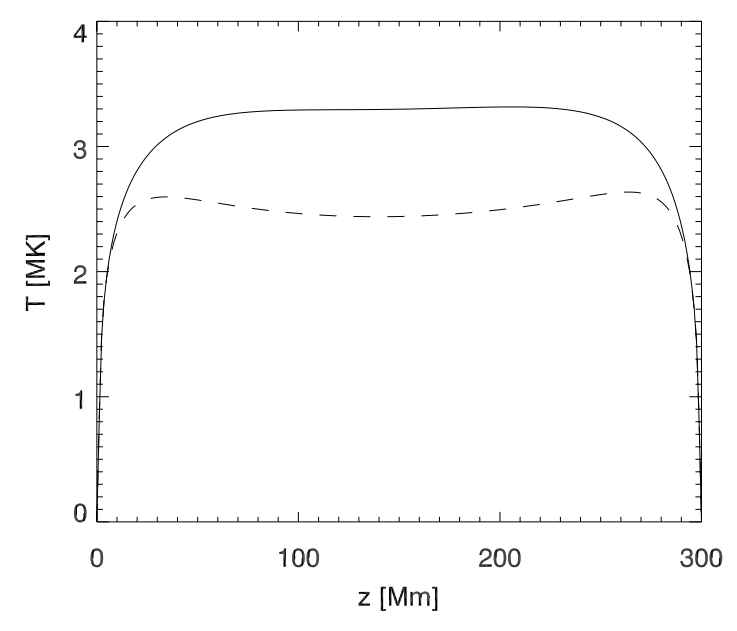

Fig. 1. Temperature along the coronal loop. Initial stable state (solid line, $H_{m}=30 \mathrm{Mm}$ ) and another stable solution for $H_{m}=12 \mathrm{Mm}$ (dashed line).

observations, while $70 \%$ of the observed loops are not compatible with hydrostatic models at all. A large set of coronal heating models has been discussed by Aschwanden (2001) in light of whether they can satisfy the observational constraints. He concludes that most of the conventional AC and DC models must be refined by including the chromosphere and transition region in order to be compatible with the observations.

\subsection{Initial state}

The coronal loop model studied here has a total length of $300 \mathrm{Mm}$, composed of a semicircular arch of $298 \mathrm{Mm}$ length and a vertical stretch of $1 \mathrm{Mm}$ length at each end. A static initial state is obtained by prescribing a large energy dissipation length of $H_{m}=30 \mathrm{Mm}$, which results in a loop apex temperature of $T=3.3 \mathrm{MK}$. The temperature along the loop of the initial state is plotted in Fig. 1.

\section{The effect of the damping length on loop evolution}

\subsection{Recurrent condensations in long loops}

The most important conclusion reached in Paper II was the fact that a thermal instability can give rise to a highly dynamic evolution of solar coronal loops. We worked out that the scale height of the energy dissipation (the so-called damping length) acts as a control parameter of this nonlinear system and determines whether there exists a stable solution. If this is not the case, thermal non-equilibrium can lead to catastrophic cooling, a self-amplifying process by which the radiative losses increase strongly over a short time scale, and result in a confined region of high-density plasma (the condensation region), which then slides down the loop legs due to gravity.

The maximum velocity of the falling condensation region is determined by its formation height and the loop plasma underneath the condensation region. We found in Paper II that the pressure of the transition region plasma can efficiently slow down the falling "blob" so that its maximum velocity may be 


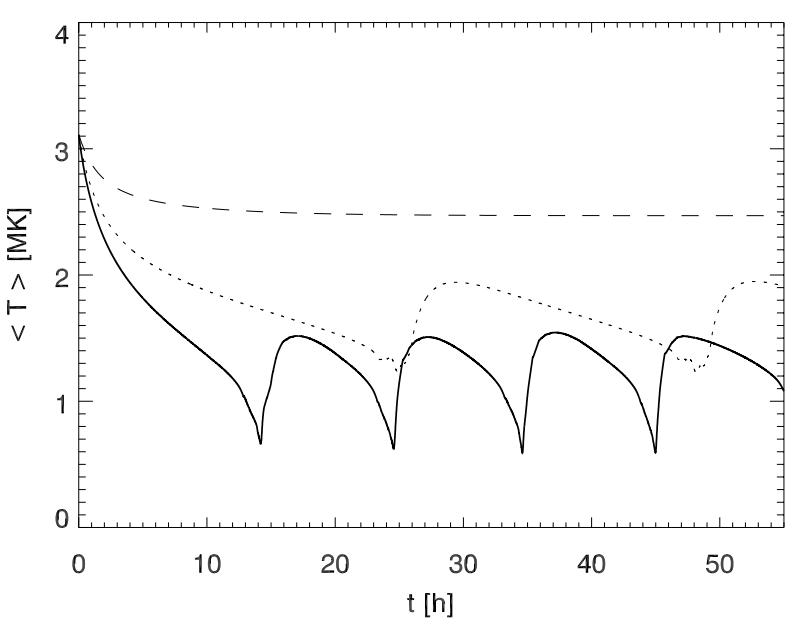

Fig. 2. Evolution of mean temperature, $\langle T\rangle$, as a function of time, for different damping lengths of the heating function: $H_{m}=2 \mathrm{Mm}$ (solid), $H_{m}=5 \mathrm{Mm}$ (dotted), and $H_{m}=12 \mathrm{Mm}$ (dashed).

substantially slower than the free-fall speed. Compared to observations of catastrophic cooling in coronal loops (Schrijver 2001; De Groof et al. 2004), the results were encouraging in the sense that the formation of such high-density regions was indeed possible without any time-dependent driving mechanism, but not fully satisfying as the deduced blob velocities of $30-40 \mathrm{~km} \mathrm{~s}^{-1}$ were lower than the observed ones (up to $100 \mathrm{~km} \mathrm{~s}^{-1}$ ).

For the new loops models with $L=300 \mathrm{Mm}$, we plot in Fig. 2 the mean temperature ${ }^{1},\langle T\rangle$, as a function of time for three different damping lengths. In agreement with the results of Paper II, we find that the period of the condensation cycle increases with increasing damping length, until finally a stable solution is reached. For damping lengths close to the limit of stability, the period of the cycle can become very long. We ran a model with $H_{m}=8 \mathrm{Mm}$ for 10 days of solar time and found a period of $P=4.15$ days.

Figure 3 shows the evolution of the mean temperature, $\langle T\rangle$, as a function of mean pressure, $\langle p\rangle$. In this plot, periodic solutions trace out cycles in $\langle p\rangle-\langle T\rangle$ space while stable solutions reach fixed points. It is seen that also in this parameter regime the loop evolution can be classified into stable solutions and dynamic solutions.

The main effects of the longer loop length and higher energy flux (with respect to the models presented in Paper II) are as follows: For a given loop length, a higher heating rate leads to a higher loop temperature and a higher density, while for a fixed energy flux of $F_{m 0}=10^{4} \mathrm{~W} \mathrm{~m}^{-2}$, the mean loop temperature of stable loops with small damping lengths $\left(H_{m} \leq 10 \mathrm{Mm}\right)$ does not depend strongly on the loop length. However, the range of damping lengths for which the loops exhibit recurrent condensations increases with loop length. For more details, the reader is referred to Müller (2004) and Müller et al. (2005).

1 We define mean values as the average quantities over the region of the loop which lies above the transition region, bounded by the points where the temperature crosses $T=10^{5} \mathrm{~K}$ in both loop legs (the exact choice of this cut-off value does not significantly influence the results and could be set to any temperature $T \gtrsim 2 \times 10^{4} \mathrm{~K}$ ).

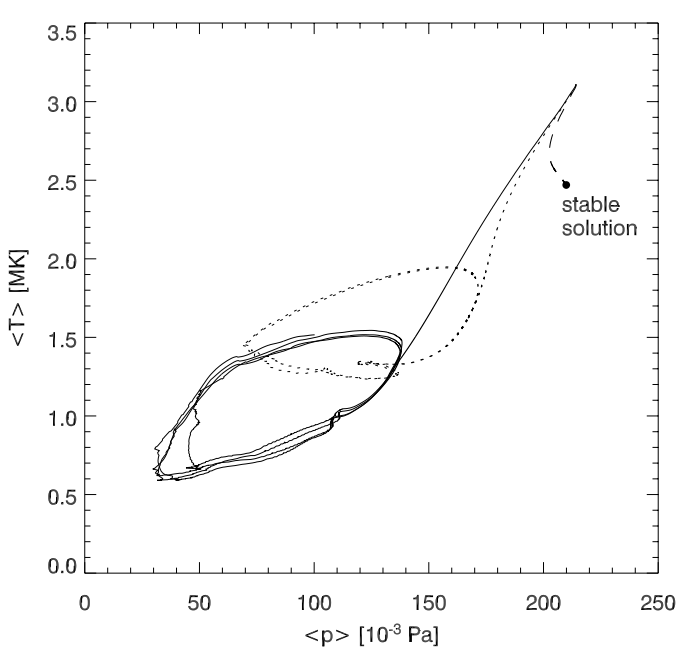

Fig. 3. Mean temperature, $\langle T\rangle$, of the loop, as a function of mean pressure, $\langle p\rangle$, for a loop of total length $L=300 \mathrm{Mm}$. Solid line: $H_{m}=2 \mathrm{Mm}$, dotted: $H_{m}=5 \mathrm{Mm}$, dashed: $H_{m}=12 \mathrm{Mm}$.

\subsection{Slow and fast blobs}

Having introduced the general characteristics of possible solutions, we now focus on the evolution of the dynamic solutions with the aim of deducing the speed of the plasma blobs which result from the catastrophic cooling process. In Fig. 4, we plot space-time diagrams of the loop temperature, $T(z, t)$, for $H_{m}=2,3,5 \mathrm{Mm}$. All three models show the recurrent condensations which have been described in detail in Paper II. We pointed out in Paper II that the highest velocities occur in the wake of a falling blob where the density is low. However, for a comparison with observations, the proper motion of the density enhancement itself has to be calculated. The maximal blob speeds for the run with $H_{m}=5 \mathrm{Mm}$ are $48 \mathrm{~km} \mathrm{~s}^{-1}$, and $42 \mathrm{~km} \mathrm{~s}^{-1}$ for the run with $H_{m}=3 \mathrm{Mm}$. This shows that the blobs are strongly decelerated on their way down since the maximal free-fall speed from the loop apex, $h_{\max }$, is $v_{\mathrm{ff}}=\sqrt{2 G m_{\odot} h_{\max } /\left(r_{\odot}\left(r_{\odot}+h_{\max }\right)\right)}=215 \mathrm{~km} \mathrm{~s}^{-1}$.

We would now like to draw the readers attention to the simulation run with $H_{m}=2 \mathrm{Mm}$. Every time after a condensation region has formed, e.g. around $t_{1}=13 \mathrm{~h}$ and $t_{2}=24 \mathrm{~h}$, a second temperature minimum occurs, and the shallow slope of its motion in the $(z, t)$-plane indicates very high speeds.

In Fig. 5 we plot the temperature for this part of the simulation, along with the velocity along the loop. The shallower slope of the solid line compared to the dashed line indicates that the second blob moves at higher speeds than the first one. Figure 6 displays the blob speed as a function of time for both the first and the second condensation region. While the first blob only reaches a velocity of around $25 \mathrm{~km} \mathrm{~s}^{-1}$, the second blob moves at a velocity of up to $90 \mathrm{~km} \mathrm{~s}^{-1}$.

While the second blob moves at up to $90 \mathrm{~km} \mathrm{~s}^{-1}$, the plasma in the wake of this blob flows at even higher speed, namely up to $230 \mathrm{~km} \mathrm{~s}^{-1}$. The fastest flows in the wake of the second blob are seen as a dark triangular structure in the velocity plot of Fig. 5. The line stretching from the left side at $t=14.2 \mathrm{~h}$ to the right side at $t=15 \mathrm{~h}$ is due to the rebound shock front which results from the blob entering the dense transition region. 


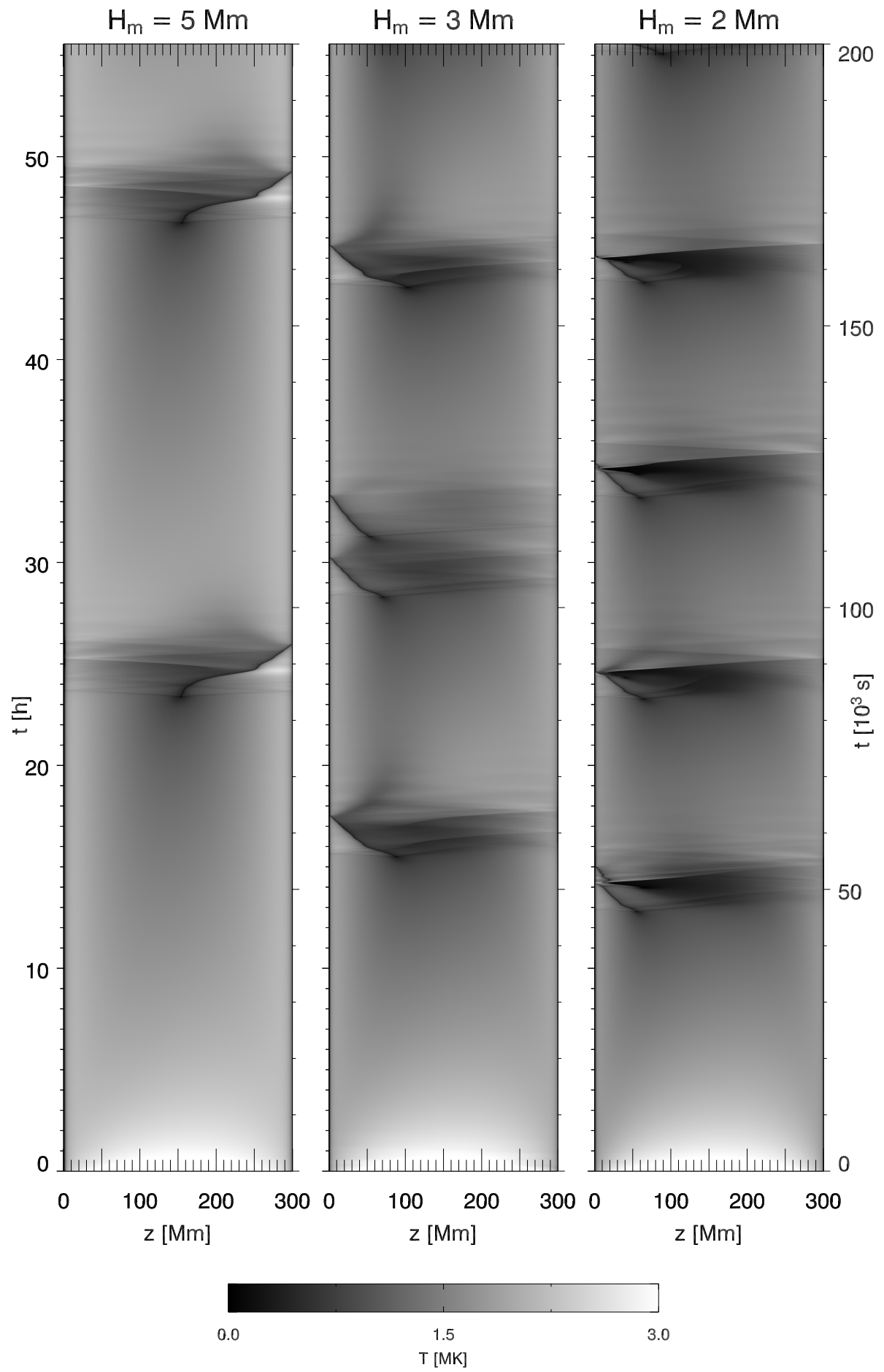

Fig. 4. Evolution of the temperature along the loop, $T(z, t)$, for three different damping lengths of the heating function: $H_{m}=5 \mathrm{Mm}(l e f t)$, $H_{m}=3 \mathrm{Mm}$ (center), $H_{m}=2 \mathrm{Mm}($ right). The loop footpoints are at $z=0$ and $300 \mathrm{Mm}$, the apex is at $z=150 \mathrm{Mm}$.

Due to the low density of this region, this shock front is not accompanied by substantial emission in any spectral line and will therefore not be discussed further.

It is seen that after a short period of nearly free fall (between $t=14.0-14.1 \mathrm{~h}$ ), the blob is strongly decelerated and even bounces upwards briefly $(t=14.2-14.3 \mathrm{~h})$. We pointed out in Paper II that in the case of a weak magnetic field the deceleration process may in reality not be as vigorous as in this one-dimensional simulation, because the enhanced pressure of such a high-density region will distort the magnetic field. However, the findings of Mackay \& Galsgaard (2001) show that a sufficiently strong magnetic field can indeed result in the dense blob rebounding several times.

\subsection{Formation of the second condensation region}

The phenomenon that a second region of enhanced density can form in the wake of another condensation region has not been 

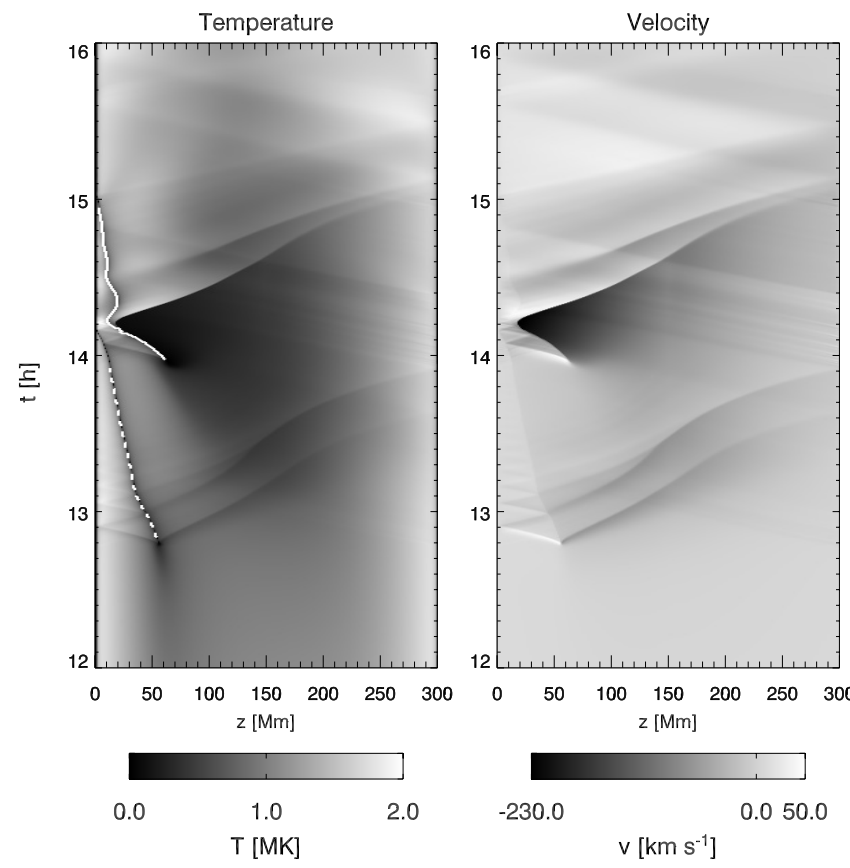

Fig. 5. Formation of the fast blob. The left plot shows the temperature $T(z, t)$ for a damping length of $H_{m}=2 \mathrm{Mm}$, the right plot displays the corresponding velocities. The dashed and the solid lines correspond to the tracks of the slow and fast blob, respectively. The corresponding blob speeds are plotted in Fig. 6 .

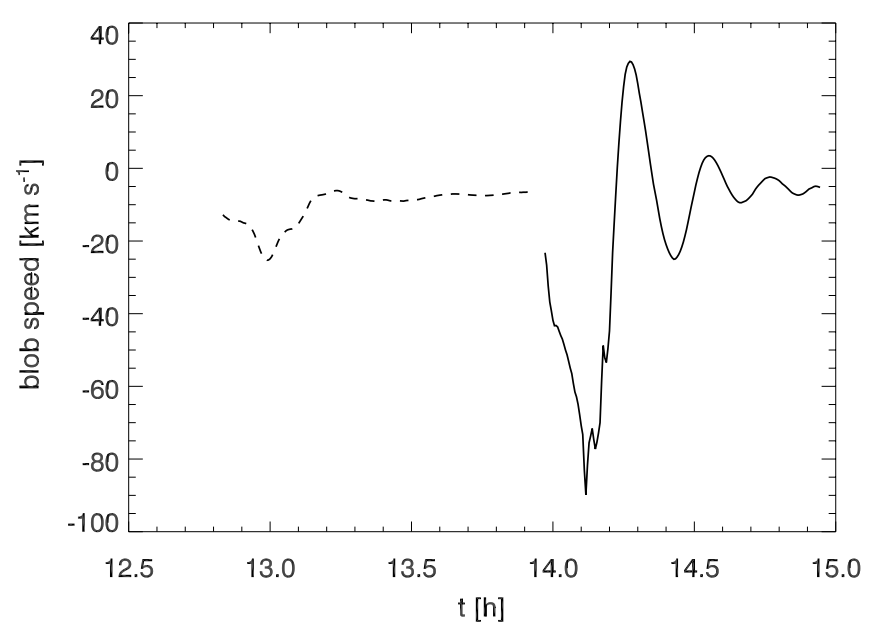

Fig. 6. Proper motion of the slow blob (dashed line) and the fast blob (solid line).

reported before and shall be explained in detail. First of all, compared to the $L=100 \mathrm{Mm}$ loops studied in Paper II with maximal apex temperatures below $7 \times 10^{5} \mathrm{~K}$, the more strongly heated $L=300 \mathrm{Mm}$ loop models studied here have apex temperatures of around $3 \mathrm{MK}$ and correspondingly higher pressures. Therefore the formation of a condensation region, where the temperature drops to around $10^{4} \mathrm{~K}$ on a very short timescale, results in a much higher pressure gradient on both sides of the condensation region. The resulting inflows towards the pressure minimum collide and produce shocks. These pressure pulses then travel away from the condensation region to both sides and lead to a transient compression of the loop plasma they encounter on their way. Even a small compression leads to a noticeable increase of the radiative losses and these radiative losses in turn give rise to enhanced cooling which results in a local density maximum. The condition for the formation of a condensation region are herewith fulfilled, and the density enhancement cools more strongly than its surroundings: catastrophic cooling sets in. In the following, this second blob is moving down the loop due to gravity while the pressure pulse (which triggered the formation of the blob) continues to propagate to the other end of the loop.

The formation of the second blob is displayed in Fig. 7: At time $t_{1}=46060 \mathrm{~s}$ (solid lines), the first condensation region has formed and appears as a narrow region with strongly decreased temperature and pressure, and strongly enhanced radiative losses and electron density. At time $t_{2}$ (dotted lines), the condensation region has traveled around $5 \mathrm{Mm}$ to the left, and a temperature and density perturbation is seen to propagate towards the loop apex. At time $t_{3}$ (dashed lines), this perturbation has led to a local maximum in the radiative losses (indicated by the right black dot and vertical lines), while the electron density is also perturbed, but does not show a local maximum yet. Finally, at time $t_{4}$ (dash-dotted lines), the second condensation sets in, and this second region of strongly decreased temperature and pressure is accompanied by local maxima of the radiative losses and electron density (indicated by the left black dot). From the separation of the peaks at $t_{3}$ and $t_{4}$ it can already be seen that the second condensation region travels much faster than the first one.

What makes this density enhancement travel so much faster than the previous one? In Fig. 8 we plot the total radiative losses (to indicate the blobs' positions) and the pressure for the slow and the fast blob for two timesteps where the blobs are at the same location $(z \approx 53 \mathrm{Mm})$. It is seen that the pressure on the left side of the leading blob is significantly higher than the one for the trailing blob. Therefore, the first blob is more strongly decelerated by the pressure of the underlying plasma, while the second one can travel in the low-pressure region in the wake of the first one.

\section{Comparison with observations}

On 11 July 2001 from 16:00 UT until 18:28 UT, an "EIT shutterless campaign" was conducted which provided 120 highcadence He II $(30.4 \mathrm{~nm})$ images of the north-eastern quarter disk of the Sun. In this observational program, which is planned every 3 months, the EIT shutter is kept open for more than 2 hours during which images are taken with a time cadence around 68 seconds. The sequence of 120 images is interrupted only by two gaps, allowing each for a LASCO C2 image in the framework of the EIT "CME watch program". Instead of the normal full disk field of view (FOV) of EIT, the shutterless program concentrates on a subfield of $416 \times 416$ pixels $\left(\approx 1082^{\prime \prime} \times 1082^{\prime \prime}\right)$, the north-eastern quarter disk in the sequence under analysis.

The most intriguing feature present in the data is an offlimb loop structure of approximately $100 \mathrm{Mm}$ height which shows intensity variations propagating downward during the whole sequence. Figure 9 displays three EIT difference images which allowed for the identification of seven propagating 

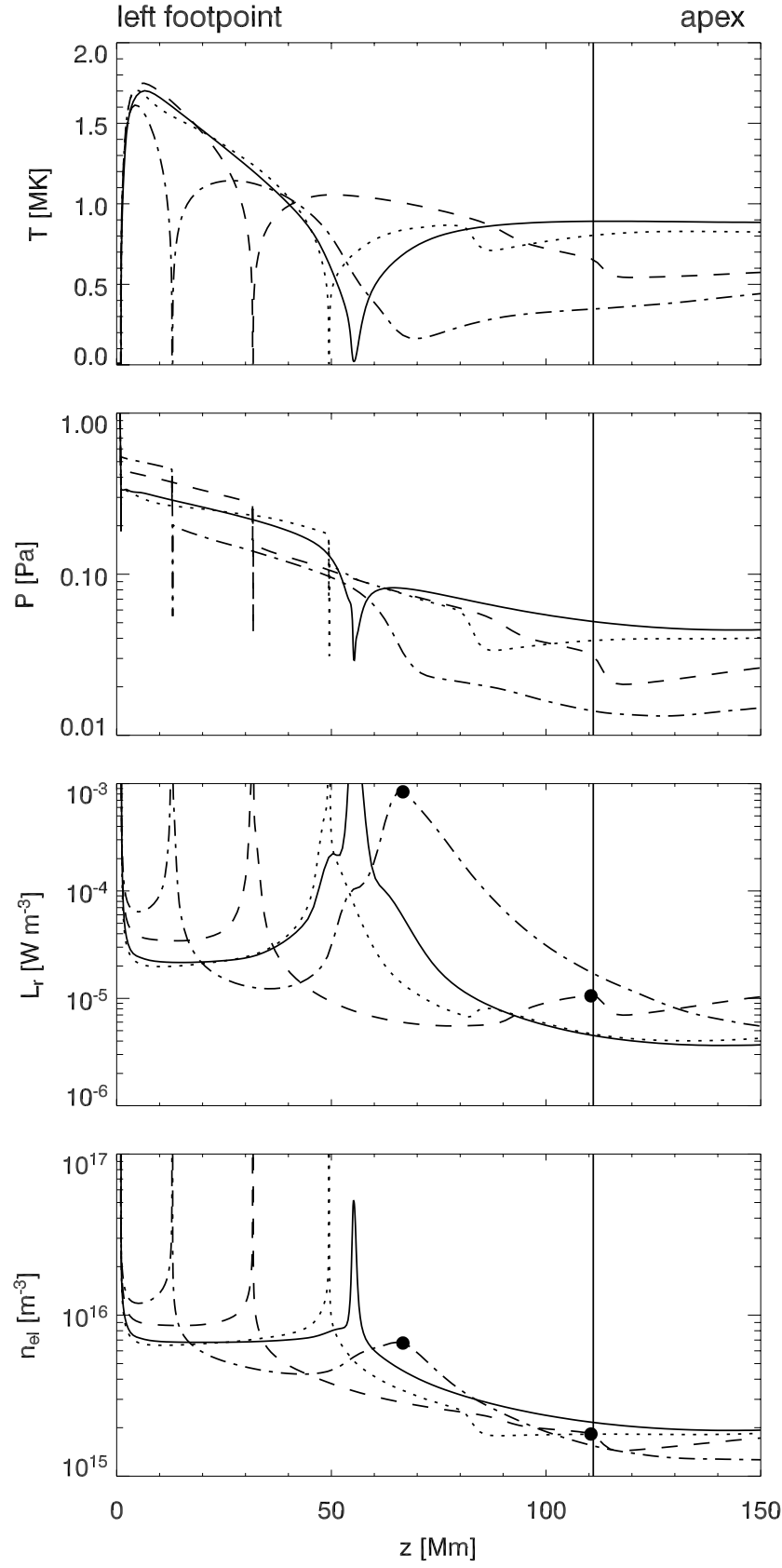

Fig. 7. Formation of the second condensation region. From top to bottom: temperature, pressure, total radiative losses and electron density along the left half of the loop for $t_{1}=46060 \mathrm{~s}$ (solid), $t_{2}=46500 \mathrm{~s}$ $($ dotted $), t_{3}=47720 \mathrm{~s}$ (dashed) and $t_{4}=50120 \mathrm{~s}$ (dash-dotted). The black dots mark the second maximum of the radiative losses which is triggered by the shock front (indicated by vertical lines) originating from the first condensation region.

intensity variations (for details, cf. De Groof et al. 2004). Since there was no evident cause noticeable in the direct neighborhood of this loop, De Groof et al. (2004) studied the characteristics of the intensity variations propagating downward in order to find out whether they could be interpreted as waves or as a flow. A multi-wavelength analysis of the time frame of the shutterless sequence revealed that the varying loop is unlikely to be flare-related (although a flare appears south of the analyzed region) and that the bright blobs consist of cool plasma
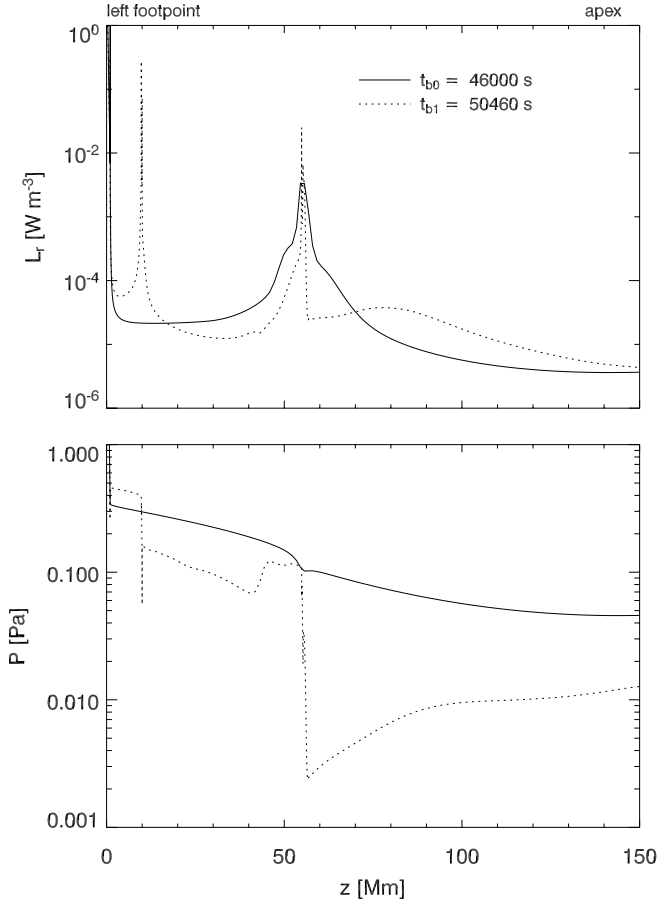

Fig. 8. Radiative losses (top) and pressure (bottom) for two time steps of the simulation. The solid line in the upper panel shows the enhanced radiation from the first blob, formed around $t_{\mathrm{b} 0}=46000 \mathrm{~s}$. The dotted line $\left(t_{\mathrm{b} 1}=50460 \mathrm{~s}\right)$ has two maxima: the one at $z=10 \mathrm{Mm}$ stems from the first blob which has moved towards the left footpoint, while the second one at $z=55 \mathrm{Mm}$ indicates the second blob. The lower panel shows that at the same location, the pressure downstream of the second blob (at $\left.t_{\mathrm{b} 1}\right)$ is lower than the pressure downstream of the first blob (at $\left.t_{\mathrm{b} 0}\right)$, which explains its higher speed.

since they are only visible in the cool temperature bands of $\mathrm{He}$ II $(30.4 \mathrm{~nm})$ and $\mathrm{H} \alpha$.

The propagating disturbances were analyzed in more detail by outlining the track along which they move down and investigating the behavior and speed of the bright blobs in a location-time diagram. Seven blobs were identified, propagating down along the loop track with speeds ranging from 30 to $120 \mathrm{~km} \mathrm{~s}^{-1}$, increasing in time (see Fig. 9). The mean intensity enhancements caused by the disturbances range from 9 to $38 \%$ or from 23 to $93 \%$ of the background intensity depending on which background subtraction is carried out.

In order to interpret the intensity variations as waves or flows, De Groof et al. (2004) compared the parameters found in the analysis with the typical parameters of slow magnetoacoustic waves observed in the solar corona. This comparison led to several anomalies, e.g. the blob speeds which are increasing and definitely higher than the local sound speed, the amplitudes which substantially exceed the intensity enhancements seen in waves and the lack of any damping. Last but not least, slow waves are only observed propagating upwards while the bright blobs we see all move downwards. On the other hand, the hypothesis of falling/flowing plasma concentrations looks more promising.

The upper panel of Fig. 10 shows the observed blob speeds as a function of height over the solar surface, as measured by De Groof et al. (2004). The different symbols correspond to the 


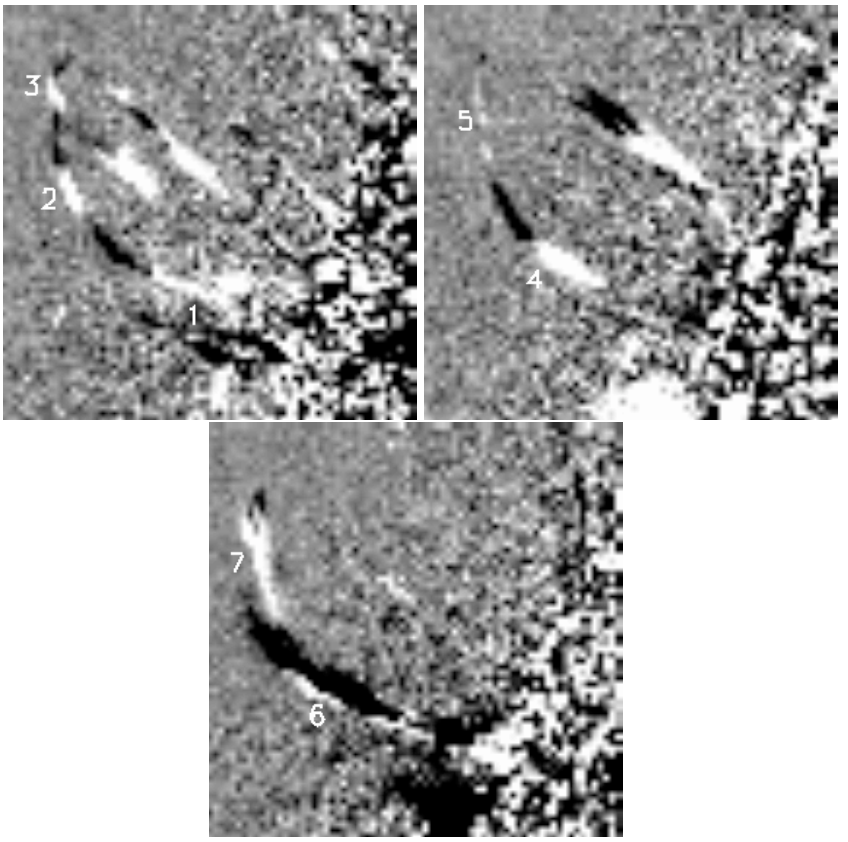

Fig. 9. Three difference images taken at 16:27, at 17:09 and at 17:58 UT, in which seven propagating blobs with enhanced intensity can be identified.

different blobs which have been identified. In the upper part of the loop, the measured speeds are closely related to the theoretical free-fall speed from a height of $100 \mathrm{Mm}$, especially when taking into account the uncertainties concerning the projection angle, the curvature of the loop and the exact loop height. In the lower part of the loop, on the other hand, the speeds clearly deviate from the free-fall curve. In the lower panel of Fig. 10 we plot the speeds of the fast blobs from our catastrophic cooling models. It turns out that the exact location where a condensation region forms depends very sensitively on the radiative losses. In the simulations where the non-equilibrium ionization of hydrogen, helium, carbon and oxygen were explicitly included in the calculations, the blobs formed at around $60 \mathrm{Mm}$ height, while they appeared at greater heights when a priori radiative loss curves as functions of electron temperature for elements other than hydrogen and helium were used. The subsequent evolution of the plasma is very similar, therefore the blob speeds for both cases are plotted in Fig. 10, together with the velocity curves for free fall from heights of $60 \mathrm{Mm}$ and $100 \mathrm{Mm}$, respectively.

Despite the fact that the accurate measurement of blob speeds is difficult, especially in the lower part of the loop, we find at least a qualitative agreement between the observed blob speeds and those derived from the model. In their early phases the blobs are accelerated to nearly free-fall speeds as the main force acting on the plasma is the field-line projected component of the solar gravitational acceleration. As the blobs fall towards the solar surface, however, the speeds deviate more and more strongly from free fall since the blob is decelerated by the underlying plasma. In contrast to the model, most of the measured blob speeds do not show a significant decrease towards the solar surface, but only a strong deviation from free-fall speed. For two blobs, indicated by the rectangles and
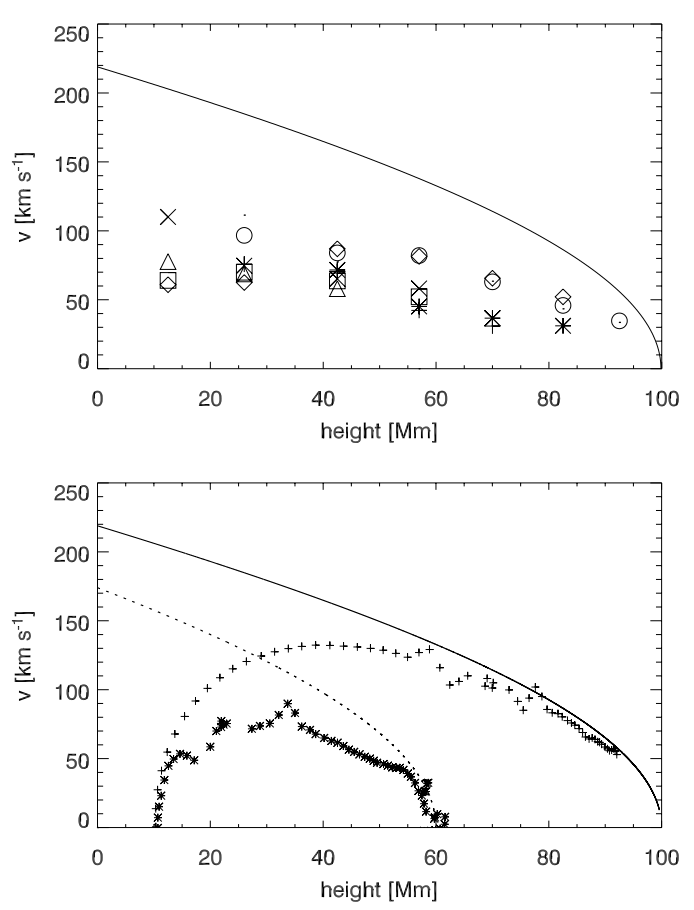

Fig. 10. Top: measured blob speeds as a function of height over the solar surface, together with the free-fall speed from a height of $100 \mathrm{Mm}$. The different symbols mark the different observed blobs. Bottom: speed of the fast blob in the simulation with $H_{m}=2 \mathrm{Mm}$. Crosses and asterisks correspond to two runs with slightly different radiative losses (cf. text). The solid line indicates a free fall from a height of $100 \mathrm{Mm}$, the dotted line corresponds to $60 \mathrm{Mm}$ height.

diamonds in Fig. 10, the speeds indeed decrease slightly in the last data points, but the number of blobs tracked is too low to make any general statement. Apart from possible shortcomings of the model, the lack of observations of decreasing speeds towards the solar limb may also be due to the fact that the loop's footpoints lie behind the limb or due to the difficulty of correct background subtraction which is described by De Groof et al. (2004). However, this is to our knowledge the first model which provides a simple physical mechanism leading to propagating intensity enhancements which reach velocities of the order of $100 \mathrm{~km} \mathrm{~s}^{-1}$.

Time-slice diagrams of the EIT data set indicate events where fast blobs catch up with blobs at lower speeds, but further analysis of more data sets is needed to confirm or reject this hypothesis. High-cadence observations of active regions above the limb in the He II $(30.4 \mathrm{~nm})$ band, $\mathrm{H} \alpha$ or C IV $(154.8 \mathrm{~nm})$ would help to shed more light onto the distribution of blob speeds as a function of height above the limb. Observations of blobs speeds which are increasing during the first phase of the fall and then decreasing when the blob approaches the solar limb would strengthen our concept of falling plasma condensations that are decelerated by the pressure of the transition region. On the other hand, observations of blobs close to the limb at speeds significantly above $100 \mathrm{~km} \mathrm{~s}^{-1}$ would indicate that the part of the loop underneath the falling blob has been previously evacuated by a different process. 


\section{Summary}

In this work, we have presented models of solar coronal loops which show catastrophic cooling events as a result of a loss of thermal equilibrium. For the first time we apply the scenario of the "heating-condensation cycle" to models of long activeregion loops and find that the shocks caused by rapid cooling processes in hot loops can trigger further cooling events. The associated condensation regions, blobs of cool plasma with enhanced density and radiative losses, can be accelerated to very high velocities.

While the leading condensation region is strongly decelerated by the underlying loop plasma, the trailing blob is traveling in the wake of the leading blob where the pressure is significantly lower. These blobs are initially traveling with almost free-fall speed and can reach velocities of the order of $100 \mathrm{~km} \mathrm{~s}^{-1}$ before their deceleration sets in in the lower regions of the atmosphere. The reason for the higher velocity of these blobs is the lower pressure in the wake of the leading blob. These fast blobs offer a possible explanation for the recent observations of propagating intensity enhancements by De Groof et al. (2004) and show that transient brightenings and high-speed downflows with a wide range of speeds can result from catastrophic cooling in coronal loops.

Acknowledgements. We thank the referee for helpful comments. This work was supported by the EU-Network HPRN-CT-2002-00310.

\section{References}

Aschwanden, M. J. 2001, ApJ, 560, 1035

Aschwanden, M. J., Nightingale, R. W., \& Alexander, D. 2000, ApJ, 541,1059

Aschwanden, M. J., Schrijver, C. J., \& Alexander, D. 2001, ApJ, 550, 1036

De Groof, A., Berghmans, D., van Driel-Gesztelyi, L., \& Poedts, S. 2004, A\&A, 415, 1141

Fredvik, T., Kjeldseth-Moe, O., Haugan, S. V. H., et al. 2002, Adv. Space Res., 30, 635

Gudiksen, B. V., \& Nordlund, Å. 2002, ApJ, 572, L113

Hansteen, V. 1993, ApJ, 402, 741

Kjeldseth-Moe, O., \& Brekke, P. 1998, Sol. Phys., 182, 73

Mackay, D. H., \& Galsgaard, K. 2001, Sol. Phys., 198, 289

Müller, D. A. N. 2004, Ph.D. Thesis, University of Freiburg http://freidok.ub.uni-freiburg.de/volltexte/1537/

Müller, D. A. N., Hansteen, V. H., \& Peter, H. 2003, A\&A, 411, 605

Müller, D. A. N., Hansteen, V. H., \& Peter, H. 2005, A\&A, in preparation

Müller, D. A. N., Peter, H., \& Hansteen, V. H. 2004, A\&A, 424, 289

Schrijver, C. J. 2001, Sol. Phys., 198, 325

Serio, S., Peres, G., Vaiana, G. S., Golub, L., \& Rosner, R. 1981, ApJ, 243, 288

Withbroe, G. L., \& Noyes, R. W. 1977, ARA\&A, 15, 363 